\title{
Metodología para la dirección de las prácticas preprofesionales en la familia de especialidades Mecánicas en la Educación Técnica Profesional*
}

\author{
Fredys García González ${ }^{\star \star}$, Vivian Arencibia Fernández ${ }^{\star \star \star}$
}

\section{RESUMEN}

Las prácticas preprofesionales en las especialidades de Mecánica en la Educación Técnica y Profesional han presentado limitaciones en la calidad de formación del egresado, cuestión motivada fundamentalmente por las deficiencias en la preparación para la dirección del sistema de influencias. De este modo, en el presente artículo se presenta una metodología para la dirección de las prácticas, la cual ha sido diseñada teniendo en cuenta las funciones de los profesores y tutores. La introducción de esta ha facilitado la preparación técnica y metodológica del personal implicado.

Palabras clave: Práctica preprofesionales, técnico en formación, tutor.

Methodology for conducting pre-professional practices in the Mechanics specialty in Professional Technical Education

\begin{abstract}
Pre-professional practices in the mechanics field at the technical and vocational education had limitations in the quality of training for graduates, due mainly to deficiencies in preparing the management of the influence system. This article presents a methodology for management practices, which was designed taking into account the role of teachers and tutors. Its introduction has facilitated the technical and methodological training of personnel involved.
\end{abstract}

Key words: Pre-professional practice, technical training, tutor.

Este artículo da cuenta de los resultados del Proyecto Nacional titulado "La formación de la fuerza laboral calificada de nivel medio. Vías para su perfeccionamiento".

* Fredys García González es Profesor Auxiliar, especialidad Mecánica de la Facultad de Ingeniería Mecánica e Industrial de la Universidad Central Marta Abreu de las Villas, Santa Clara, Cuba. E-mail: fredysg@uclv.cu

Vivian Arencibia Fernández es Profesora Auxiliar, especialidad Construcción de la Facultad Construcciones de la Universidad Central Marta Abreu de las Villas, Santa Clara, Cuba. E-mail: viviana@uclv.cu 


\section{Introducción}

La escuela constituye el contexto educativo esencial en la que ocurre la formación técnico-profesional de la fuerza laboral competente. Así, tiene este encargo social y contribuye al desarrollo pleno de la personalidad del estudiante y a la preparación como ciudadano independiente y transformador del medio social en las condiciones históricas que le ha tocado vivir. Al estudiante se le ha de preparar para enfrentar con éxito las exigencias del nivel educativo y de la sociedad en que vive. Acerca de esto, Martí (I883, p. 53) expresa: "(...) En la escuela se ha de aprender el manejo de las fuerzas con que en la vida se ha de luchar".

En la Educación Técnica y Profesional (ETP) esto conduce a la necesidad de integración de diferentes contextos educativos para lograr la formación integral del estudiante, en este caso, técnico en formación. Esta visión implica asumir la práctica educativa diferente y colocar en el centro del proceso al técnico en formación $y$, a su vez, analizar sus relaciones con otros contextos educativos para comprender su comportamiento y guiar su formación técnicoprofesional integral.

La integración de la escuela con otros contextos educativos ha sido analizada por varios autores en la literatura pedagógica, entre los que se pueden mencionar a Chávez, Suárez y Permuy (2005). Sobre este punto, los autores sostiene que "la escuela tiene un encargo social que cumplir, pero no puede hacerlo en forma autoritaria, por lo que debe buscar siempre la amplia participación de todo el colectivo en la toma de decisiones por medio del debate activo y democrático" (Chávez, Suárez y Permuy, 2005, p. 59). En la escuela politécnica este colectivo trasciende los marcos del contexto escolar e incluye a la entidad laboral, a la familia y a la comunidad.

La entidad laboral es uno de los contextos educativos que influye decisivamente en la formación del futuro Técnico Medio. Esta pertenece al mundo laboral, en el cual se inserta al técnico en formación de la escuela politécnica durante el periodo de realización de las prácticas preprofesionales. Así, se debe aclarar lo que sigue: 
Las prácticas preprofesionales constituyen la modalidad de enseñanza práctica que consiste en vincular a tiempo completo a los estudiantes de nivel técnico medio a centros de producción o servicios que reúnan las condiciones técnicas-organizativas y tecnológicas necesarias para realizar la práctica integral dirigida a la profundización de los conocimientos, habilidades, hábitos y valores laborales adquiridos en el periodo de formación del profesional, atendidos por tutores de las entidades laborales y profesores de los centros docentes (MINED, 20I3, p. 36).

Desde las vivencias como investigadores, la participación en inspecciones y entrenamientos metodológicos conjuntamente con la Dirección Provincial de Educación, resultados de visitas especializadas a las entidades laborales, encuestas y entrevistas con profesores y tutores que dirigen las prácticas profesionales del Técnico Medio en la familia de especialidades de Mecánica, se han podido constatar las siguientes regularidades:

I. Se evidencia en más de un 50\% que los técnicos en formación tienen dificultades con el dominio de las habilidades básicas de la especialidad durante el desarrollo de las prácticas preprofesionales.

2. La mayoría de los egresados muestran baja preparación profesional que se manifiesta en la poca diversidad de problemas profesionales de la especialidad que pueden resolver.

3. Falta de cobertura e idoneidad de los profesores, reconociéndose que el claustro de las prácticas preprofesionales tiene carencias en la aplicación de la metodología de la enseñanza práctica y/o no tienen experiencia técnica suficiente lo que incide desfavorablemente en el técnico en formación.

4. Inestabilidad en el personal educativo (profesores, especialistas y tutores) de las prácticas preprofesionales por razones motivacionales y organizativas.

5. Insuficiente preparación metodológica de los tutores y tecnológica de los profesores responsables para la dirección de las prácticas preprofesionales. 
6. La preparación técnica y metodológica que estos docentes y tutores reciben del sistema de influencias no cubre todas las expectativas de necesidades para el cumplimiento de los objetivos del año y del plan de estudio de la especialidad.

Como resultado de este análisis, fueron consultadas investigaciones de postgrado que abordan la temática, entre ellos: "Estrategia metodológica para la preparación de los tutores de las prácticas preprofesionales en la especialidad de Construcción Civil" (Alonso, 2009) y "Modelo de capacitación a tutores de las entidades laborales en la especialidad de contabilidad en la Educación Técnica y Profesional” (Rodríguez, 20II).

Las referencias consultadas muestran avances referidos al tema en la ETP, sin embargo, aún se adolece de los sustentos teóricos y resultados prácticos referidos a las prácticas preprofesionales en el contexto de las especialidades de Mecánica, permitiendo al autor asumir el criterio de contribuir a la integración entre la escuela politécnica y la entidad laboral sobre la base del sólido ideario pedagógico martiano que expresa: "(...) No fructifica la educación si no es continua y constante” (Martí, I975, p. 62). De este modo, se propone una metodología que en este contexto se requiere en la dirección de las prácticas preprofesionales en las especialidades de Mecánica y se revela la necesidad de continuar perfeccionando este subsistema educacional mediante la búsqueda de alternativas que garanticen la elevación constante de su calidad.

\section{Metodología para la dirección de las prácticas preprofesionales}

La propuesta de la metodología parte de las vivencias como investigador en la temática y evoluciona enriqueciéndola con criterios de profesores que atienden las prácticas en los especialidades de Mecánica en las escuelas politécnicas, profesores de la Universidad de Ciencias Pedagógicas Félix Varela Morales, experiencias en la tutoría en las entidades laborales de la provincia Villa Clara y se diseña a partir del desarrollo de diferentes etapas, a saber: 
- Etapa I. Preparación a los profesores y tutores.

- Etapa II. Caracterización de las entidades laborales.

- Etapa III. Planificación de las prácticas preprofesionales.

- Etapa IV. Ejecución de las prácticas preprofesionales.

- Momento I: Familiarización con la entidad laboral y con el puesto de trabajo.

- Momento II: Desarrollo dirigido al tratamiento de los problemas profesionales y a la consolidación de los conocimientos, habilidades y hábitos básicos de la especialidad.

- Momento III: Evaluación de los resultados en la ejecución de la práctica.

- Etapa V. Evaluación final y conclusiones de las prácticas preprofesionales.

A continuación, se describe cada una de las etapas señaladas.

\section{Etapa I. Preparación a los profesores que dirigen las prácticas y a los tutores}

Para enfrentar el trabajo de integración entre la escuela y las entidades productivas en cada comunidad, la preparación de todos los profesionales y directivos es básica, puesto que el profesor tiene que conocer los distintos elementos de la entidad productiva para poder orientar en forma adecuada a los técnicos en formación, así como a los trabajadores que fungirán como instructores y tutores.

En esta etapa se prepara a los encargados de planificar, organizar, orientar, controlar y evaluar el desarrollo de la práctica, es decir, a los profesores de las prácticas preprofesionales de las especialidades de la familia de Mecánica y tutores e instructores implicados de la entidad laboral con el objetivo de interpretar la metodología para la organización de las prácticas preprofesionales.

La formación permanente del personal pedagógico incluye: la superación, el trabajo metodológico y la actividad científica investigativa que realiza como profesor. Estas vías deben constituir un sistema para lograr el efecto en la preparación del personal. 
La rotación por puestos de trabajo de las entidades laborales, los cursos de superación, entrenamientos, diplomados, maestrías y doctorados son variantes de superación; las actividades metodológicas que se realizan en las escuelas politécnicas, sobre todo, las dirigidas por el subdirector de enseñanza práctica y producción constituyen una vía esencial para la preparación didáctica de los profesores de las prácticas preprofesionales, y la realización de investigaciones, su socialización e introducción en la práctica educativa son una tercera vía que garantiza la formación permanente del personal docente, vinculada con la práctica en la entidad laboral.

Ahora bien, el sistema de preparación de los profesores se concibe tipo curso (96 horas), a partir de un primer módulo básico dictado en la modalidad de postgrado, denominado Enseñanza Práctica en la Escuela Politécnica (Anexo l). Esta preparación técnica metodológica con un fondo de tiempo de 48 horas está dirigida a la actualización y profundización de conocimientos relacionados con la enseñanza práctica. En un segundo momento, se proponen cursos optativos (Anexo 2), con un fondo de tiempo de 48 horas cada uno, que complementan la formación específica en correspondencia con los talleres y puestos de trabajo por los que rotan los técnicos en formación en dependencia de la especialidad.

Se plantea a través de la autopreparación de los docentes, el dominio de las resoluciones y documentos normativos que regulan los convenios y la estrategia para el cumplimiento de los mismos en las entidades laborales del territorio, implicando a las dos instituciones formativas: la escuela politécnica y la entidad laboral, de tal forma que se asegure la atención profesional formadora del educando.

Pero si importante es la labor del docente, también, lo es la de los instructores y directivos de la entidad productiva. El trabajo del instructor de la entidad productiva cumple objetivos de carácter político-ideológico, de carácter pedagógico y de carácter social, de ahí que sea vital la preparación del instructor para las tareas con los estudiantes.

Esta actividad exige una sólida preparación en el aspecto técnico y la incorporación de aspectos básicos del trabajo pedagógico que 
le permitan comunicarse adecuadamente con los estudiantes para transmitir los conocimientos, habilidades, destrezas y experiencias.

Para ello, se asume la estrategia de perfeccionamiento de la preparación de los instructores de las entidades productivas que forman parte del Modelo de Escuela Politécnica Cubana abordado por León (2003). La estrategia consta de los siguientes pasos:

a) Seleccionar a los trabajadores instructores de las empresas. Deben ser operarios con una preparación profesional adecuada y con resultados destacados en su trabajo, con experiencia profesional de varios años de trabajo y que hayan mantenido un elevado cumplimiento en los índices que miden eficiencia y actitud consecuente ante las tareas del centro. Además, deben contar con cualidades morales que les permitan educar a los estudiantes.

b) Incorporar a los instructores a las diferentes vías de superación del Sistema Nacional de Educación, a los centros de capacitación de las empresas, en las diversas modalidades como cursos desde el puesto de trabajo, postgrados, cursos de superación a tiempo completo o reciclaje en centros docentes como profesores de asignaturas de enseñanza práctica.

c) Integrar el trabajo del docente de la ETP. Esto se puede hacer a través de los entrenamientos metodológicos conjuntos; participación en la elaboración de clases y en el análisis de grupos y asambleas de padres.

d) Garantizar el reconocimiento moral por el trabajo realizado. Fundamentalmente, por medio de reconocimiento ante el colectivo laboral, distinciones y otras formas de estimulación.

e) Incentivar la participación de los instructores en los eventos científicos. Por ejemplo, pueden ser jornadas pedagógicas, fórum de Ciencia y Técnica o sociedades científicas con los estudiantes.

f) Garantizar que el instructor conozca el potencial educativo de la entidad productiva para lograr una mayor influencia en la transformación de los estudiantes. 
La preparación a los tutores debe ser ejecutada por los profesores de las prácticas preprofesionales, pues están más cerca de los tutores durante la formación profesional. También, pueden ser invitados otros docentes del colectivo de Departamento. Su ejecución es posible tanto en la escuela politécnica como en la entidad laboral, y se sugiere usar la segunda, de modo que no salga de la entidad por un tiempo prolongado. De hecho, se debe aprovechar el tiempo de capacitación que tienen previsto en su contrato de trabajo. Las vías a utilizar deben ser las acordadas en los convenios de integración escuela politécnicaentidad laboral, tales como: despachos, visitas y encuentros.

En esta etapa se realiza la preparación básica en función de la metodología a seguir durante la actividad, de manera que se pueda determinar y establecer la interrelación entre demanda social, objeto de la profesión, problema profesional, objetivo, forma de organización y evaluación. Cabe señalar que se debe prestarse atención a las relaciones entre los sujetos que participan, es decir, técnico en formación, grupo estudiantil, obreros, profesor y tutor.

Además, esta etapa comprende proponer el análisis de la documentación tecnológica a utilizar en las actividades productivas, la cual incluye los planos de piezas, las normas técnicas, los precios de la materia prima, los manuales de uso de las máquinas herramientas y de los equipos, las especificaciones técnicas, los informes técnicos de las revisiones. Es relevante considerar el mantenimiento periódico que debe ejecutar el Departamento de Normalización, Metrología y Control de la Calidad, u otro responsabilizado con esta tarea.

Por último, la evaluación de los resultados debe concluir con la categorización del tutor, la cual podría ser como docente adjunto de la escuela politécnica, cuando se trata de profesionales con nivel superior universitario. Así, la preparación se convierte en un estímulo para el profesional.

\section{Etapa II. Caracterización de las entidades laborales}

La caracterización permite determinar las fortalezas y debilidades que presentan las entidades laborales seleccionadas para poder 
planificar el sistema de actividades a desarrollar por los técnicos en formación. Asimismo, facilita adecuar la ubicación de los estudiantes a partir del diagnóstico, teniendo en cuenda las condiciones objetivas de las entidades laborales. También, ayuda a analizar el nivel de correspondencias con los intereses del perfil ocupacional y plan de estudio, entre otros.

La caracterización de las entidades laborales debe incluir datos generales y datos específicos. Esta se puede profundizar con la propia participación de los técnicos en formación durante las primeras semanas de la práctica, lo que constituye una manera de aprovechar el componente investigativo de los educandos. La información que se recolecta debe referirse a la historia de la entidad laboral, descripción de trabajadores, producción y servicio que ofrece la entidad e integración política de sus trabajadores.

\section{Etapa III. Planificación de las prácticas preprofesionales}

Los convenios que se establecen entre las escuelas politécnicas y los de producción, servicios o investigación para el desarrollo de la enseñanza práctica constituyen unos de los primeros aspectos en la planificación y contemplan, entre otros, los aspectos esenciales siguientes: cantidad de técnicos en formación que realizarán cada una de las actividades, puestos de trabajo en que se ubicarán, contenido de trabajo que desarrollarán, tiempo que durará cada actividad, horario de trabajo, precepto de protección e higiene del trabajo y normas técnicas que han de cumplirse, atención que se brindará a los educandos, nombre y funciones de los compañeros que se responsabilizan con cada actividad por ambas partes.

\section{Diseño del plan de actividades de los técnicos en formación}

El diseño es realizado por el profesor antes de la inserción de los técnicos en formación en las prácticas con la participación de estos, ajustándolo a las condiciones específicas del centro de producción, servicios o investigación donde se realizará la práctica. 
El plan constituye la guía que el técnico en formación con ayuda del tutor cumplirán durante el periodo de las prácticas. Este incluye las tareas específicas que se deben desarrollar, permitiendo el aprendizaje del educando y la actividad productiva en la entidad laboral. El plan de actividades puede estar sujeto a cambio según las condiciones concretas y, sobre todo, en relación al trabajo del tutor.

\section{Reunión de orientación de las prácticas preprofesionales}

Antes de iniciar la práctica preprofesional se efectúa una reunión con los técnicos en formación, padres y profesores con el objetivo de informar las características en que se desarrollará la misma, incluyendo la presentación de los docentes, de los centros en que serán ubicados y la definición de los horarios de prácticas. Al mismo tiempo, se realiza una explicación sobre el sistema de control y evaluación.

\section{Etapa IV. Ejecución de las prácticas preprofesionales}

Esta etapa es donde los técnicos en formación tienen el papel protagónico en el cumplimiento del plan de actividades. Se divide en dos momentos importantes: el trabajo individual a través de la tutoría y el trabajo grupal en los talleres.

\section{Momento I: Familiarización con la entidad laboral y con el puesto de trabajo}

Este momento tiene el propósito de familiarizar a los técnicos en formación con el mundo laboral. De esta manera, se presupone que el técnico en formación vaya conociendo el nuevo ambiente en el cual se inserta. Así, se realiza un recibimiento por medio de una reunión informativa sobre la entidad laboral, un breve recorrido por sus instalaciones, un encuentro con los dirigentes del taller, con los obreros de vanguardia y con los graduados de las escuelas 
politécnicas. En este contacto, la dirección de la entidad laboral da a conocer la cantidad de trabajadores, el objeto social, significado para la economía nacional, y se contarán las tradiciones de la entidad, exhortando a su continuación. También, se entrega información relativa al modo de funcionamiento, las áreas, operaciones y tareas de la producción o servicios a los que se enfrentan los técnicos, así como los materiales y productos utilizados. A través de esta acción se busca sensibilizar a los técnicos en formación sobre las normas técnicas de las operaciones y respecto de las medidas de seguridad y protección del trabajo para la protección del medio ambiente y para la salud del hombre como productor de bienes materiales, desde el propio puesto de trabajo, considerando este sitio como la célula fundamental del proceso productivo.

\section{Momento II: Desarrollo dirigido al tratamiento de los problemas profesionales y a la consolidación de los conocimientos, habilidades y hábitos básicos de la especialidad}

Este momento contempla mayor tiempo, dado que los técnicos en formación realizan trabajos individuales en sus puestos laborales. En este punto, se pretende consolidar habilidades básicas de la especialidad, que fueron desarrolladas en las áreas docentes productivas en la escuela politécnica y en los talleres productivos docentes en la entidad laboral, a partir de la solución de problemas profesionales simples, tales como: interpretar planos y croquis, medir con diferentes instrumentos, trazar, cortar con herramientas manuales y mecánicas, seleccionar materiales, operar herramientas, dispositivos y máquinas, etc. Además, se considera forjar las habilidades específicas destinadas a la resolución de los problemas profesionales de mediana y determinada complejidad en la entidad laboral.

Este momento incluye, también, trabajo grupal. Para ello, se convocan talleres con el objetivo de socializar en el colectivo las experiencias alcanzadas en los puestos de trabajo y debatir sobre los obstáculos presentados y las soluciones propuestas. 


\section{Momento III: Evaluación de los resultados en la ejecución de la práctica}

Este momento se dirige a la constatación del grado de cumplimiento o acercamiento al objetivo. En su sentido más amplio, comprende el grado de respuesta que el resultado da al proceso como un todo, o sea, en correspondencia con la demanda social al objeto de la profesión y al problema profesional. De esta manera, se evalúa el proceso en sus diversas dimensiones y se puede determinar en qué medida se lograron los objetivos propuestos. Este acto constituye un balance de las etapas y momentos descritos. En el fondo, se controla el cumplimiento del plan de actividades ejecutado por los técnicos en formación en las entidades laborales, así como el plan de rotación en función del perfil ocupacional. Además, se evalúa el desempeño de los educandos en función de aspectos formativos como: disciplina laboral, laboriosidad, honestidad, organización del puesto de trabajo, cumplimiento de las normas establecidas de calidad, higiene, protección y otras actividades de carácter político-ideológico.

Al finalizar este momento, se efectúa una evaluación parcial y se emiten los criterios correspondientes por parte del tutor y el profesor de las prácticas. Por cierto, se deben tener en cuenta los juicios valorativos de los técnicos en formación.

\section{Etapa V. Evaluación final y conclusiones de las prácticas preprofesionales}

El objetivo fundamental de esta etapa es que los técnicos en formación presenten los resultados del trabajo realizado, las experiencias acumuladas, el cumplimiento del plan de actividades, las principales evidencias en las actividades desarrolladas, así como los problemas que tuvieron y las soluciones que lograron encontrar.

En la última semana de la práctica preprofesional, cada técnico en formación debe elaborar un pre-informe sobre el trabajo efectuado, las observaciones de carácter técnico, organizativo y laboral, y recomendaciones pertinentes. Asimismo, en esta etapa 
se confeccionan posters, láminas y maquetas para la explicación y demostración del informe final.

Por último, es importante recalcar que tienen lugar eventos de los informes finales, exponiendo los resultados ante un tribunal integrado por dirigentes de la entidad laboral y la escuela politécnica, profesores y tutores. En este acto se formularán las preguntas necesarias a los técnicos en formación y se darán los resultados del desarrollo individual alcanzado en las prácticas.

\section{A modo de cierre}

Para concluir se debe decir, en primer lugar, que la metodología propuesta para la preparación técnica y didáctica de los profesores de las prácticas preprofesionales tiene en cuenta las funciones de los docentes y tutores de las entidades laborales y las diferentes etapas que intervienen en función de la organización, planificación, ejecución, control y evaluación.

En segundo lugar, con su implementación se constata que la labor profesional del profesor de las prácticas y del tutor en el vínculo de integración entre la Escuela Politécnica y Entidad Laboral permite lograr la formación integral profesional del Técnico Medio en las especialidades de Mecánica. En efecto, esto apunta al desarrollo de una formación integral de los educandos a partir de su propio desempeño como practicante en el mundo laboral.

\section{Referencias bibliográficas}

Alonso, O. (2009). Estrategia metodológica para la preparación de los tutores de las prácticas preprofesionales en la especialidad de Construcción Civil (Tesis para optar al grado de Máster en Ciencias de la Educación). Universidad de Ciencias Pedagógicas Félix Varela Morales, Villa Clara, Cuba.

Chávez, J., Suárez, A. Permuy, L. (2005). Acercamiento necesario a la pedagogía general. La Habana: Pueblo y Educación.

León, M. (2003). Modelo teórico para la integración escuela politécnica - mundo laboral en la formación de profesionales de nivel medio (Tesis para optar al grado de Doctor en Ciencias Pedagógicas). Instituto Superior Pedagógico Enrique José Varona, La Habana, Cuba. 


\section{Referencias bibliográficas}

Martí, J. (1975). Obras completas. La Habana: Ciencias Sociales.

MINED (2013). Resolución Ministerial No. 254/2013. Reglamento para la planificación, organización, desarrollo y control de la enseñanza práctica en los centros docentes de la Educación Técnica y Profesional y en las entidades de la producción y los servicios. Ciudad de La Habana: MINED.

Rodríguez, M. C. (20II). Modelo de capacitación a tutores de las entidades laborales en la especialidad de contabilidad en la Educación Técnica y Profesional. (Tesis para optar al grado de Doctor en Ciencias Pedagógicas). Universidad de Ciencias Pedagógicas Félix Varela Morales, Villa Clara, Cuba.

\section{Anexo 1}

\section{Plan temático}

\begin{tabular}{|c|l|c|c|}
\hline TEMAS & \multicolumn{1}{|c|}{ TÍTULO Y CONTENIDOS } & \multicolumn{1}{|c|}{$\begin{array}{c}\text { HORAS } \\
\text { PRESENCIALES }\end{array}$} & $\begin{array}{c}\text { HORAS } \\
\text { INTERPRESENCIALES }\end{array}$ \\
\hline I & $\begin{array}{l}\text { La Enseñanza Práctica. } \\
\text { Generalidades de la enseñanza } \\
\text { práctica. } \\
\text { Modalidades de la enseñanza } \\
\text { práctica. } \\
\text { Planificación y organización } \\
\text { de la enseñanza práctica }\end{array}$ & 6 & 2 \\
\hline 2 & $\begin{array}{l}\text { Integración Escuela } \\
\text { Politécnica - Entidades } \\
\text { laborales - comunidad. } \\
\text { Aprendizaje en la integración } \\
\text { Escuela Politécnica - Entidad } \\
\text { laboral - Comunidad. } \\
\text { Condiciones para la } \\
\text { organización de la integración } \\
\text { Escuela Politécnica - Entidad } \\
\text { Productiva - Comunidad }\end{array}$ & 6 & \\
\hline 3 & $\begin{array}{l}\text { Los convenios de trabajo } \\
\text { entre la Escuela Politécnica y } \\
\text { Entidad Productiva } \\
\text { Antecedentes y } \\
\text { particularidades } \\
\text { Características, estructura y } \\
\text { contenidos de los convenios } \\
\text { de trabajos }\end{array}$ & 2 \\
\hline
\end{tabular}




\begin{tabular}{|c|c|c|c|}
\hline 4 & $\begin{array}{l}\text { Las aulas anexas } \\
\text { Planificación y organización } \\
\text { de las aulas anexas. } \\
\text { Evaluación del proceso de } \\
\text { enseñanza aprendizaje en las } \\
\text { aulas anexas }\end{array}$ & 6 & 2 \\
\hline 5 & $\begin{array}{l}\text { La práctica Preprofesional } \\
\text { Atención a los técnicos en } \\
\text { formación en las prácticas } \\
\text { preprofesionales } \\
\text { Consideraciones } \\
\text { metodológicas para la } \\
\text { dirección de las prácticas } \\
\text { preprofesionales en la familia } \\
\text { de especialidades de Mecánica } \\
\text { en la Educación Técnica y } \\
\text { Profesional } \\
\text { Indicadores a tener en } \\
\text { cuenta para la evaluación } \\
\text { de los trabajos prácticos } \\
\text { desarrollados en las prácticas } \\
\text { preprofesionales }\end{array}$ & 6 & 2 \\
\hline 6 & $\begin{array}{l}\text { La tarea integradora } \\
\text { Planificación y organización } \\
\text { de la tarea integradora } \\
\text { Características de la tarea } \\
\text { integradora } \\
\text { Particularidades que } \\
\text { distinguen a la tarea } \\
\text { integradora }\end{array}$ & 6 & 2 \\
\hline & Total & 36 & 12 \\
\hline & Totales & \multicolumn{2}{|c|}{48} \\
\hline
\end{tabular}

\section{Anexo 2}

Las asignaturas optativas se proponen en dependencias de las especialidades de la familia de Mecánica.

I. Asignaturas de postgrados optativas:

2. Tecnología y práctica de Metalurgia ferrosa y no ferrosa. (48 horas)

3. Tecnología y práctica de Conformación de metales. (48 horas)

4. Tecnología y práctica de Normalización, Metrología y Calidad. (48 horas) 
Metodología para la dirección de las prácticas preprofesionales en la familia - García, Arancibia

5. Tecnología y práctica de Construcción de Estructuras. (48 horas)

6. Tecnología y práctica de Mecánica Industrial. (48 horas) 